ARTICLE

\title{
Robust growth of two-dimensional metal dichalcogenides and their alloys by active chalcogen monomer supply
}

\author{
Yonggang Zuo (1) 1,2,3,12, Can Liu (1) 1,4,12凶, Liping Ding5,12, Ruixi Qiao6,12, Jinpeng Tian (1) 2 , Chang Liu6 \\ Qinghe Wang ${ }^{1}$, Guodong Xue ${ }^{1}$, Yilong You${ }^{1}$, Quanlin Guo ${ }^{1}$, Jinhuan Wang ${ }^{1}$, Ying Fu ${ }^{7}$ Kehai Liu ${ }^{7}$, Xu Zhou (1) ${ }^{8}$,

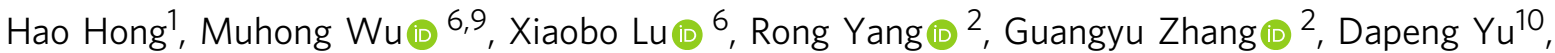 \\ Enge Wang $6,7,11$, Xuedong Bai (1) ${ }^{2,7 凶}$, Feng Ding (iD ${ }^{5 凶} \&$ Kaihui Liu (1) $1,6,7 凶$
}

The precise precursor supply is a precondition for controllable growth of two-dimensional (2D) transition metal dichalcogenides (TMDs). Although great efforts have been devoted to modulating the transition metal supply, few effective methods of chalcogen feeding control were developed. Here we report a strategy of using active chalcogen monomer supply to grow high-quality TMDs in a robust and controllable manner, e.g., $\mathrm{MoS}_{2}$ monolayers perform representative photoluminescent circular helicity of $\sim 92 \%$ and electronic mobility of $\sim 42$ $\mathrm{cm}^{2} \mathrm{~V}^{-1} \mathrm{~s}^{-1}$. Meanwhile, a uniform quaternary TMD alloy with three different anions, i.e., $\mathrm{MoS}_{2(1-x-y)} \mathrm{Se}_{2 x} \mathrm{Te}_{2 y}$, was accomplished. Our mechanism study revealed that the active chalcogen monomers can bind and diffuse freely on a TMD surface, which enables the effective nucleation, reaction, vacancy healing and alloy formation during the growth. Our work offers a degree of freedom for the controllable synthesis of 2D compounds and their alloys, benefiting the development of high-end devices with desired 2D materials.

\footnotetext{
${ }^{1}$ State Key Laboratory for Mesoscopic Physics, Frontiers Science Centre for Nano-optoelectronics, School of Physics, Peking University, 100871 Beijing, China. ${ }^{2}$ Beijing National Laboratory for Condensed Matter Physics, Institute of Physics, Chinese Academy of Sciences, 100190 Beijing, China. ${ }^{3}$ The Key Laboratory of Unconventional Metallurgy, Ministry of Education, Faculty of Metallurgical and Energy Engineering, Kunming University of Science and Technology, Kunming 650093 Yunnan, China. ${ }^{4}$ Department of Physics and Beijing Key Laboratory of Opto-electronic Functional Materials\&Micro-nano Devices, Renmin University of China, 100872 Beijing, China. ${ }^{5}$ Centre for Multidimensional Carbon Materials, Institute for Basic Science, Ulsan 44919, South Korea.

${ }^{6}$ International Centre for Quantum Materials, Collaborative Innovation Center of Quantum Matter, Peking University, 100871 Beijing, China. ${ }^{7}$ Songshan Lake Materials Laboratory, Dongguan 523808 Guangdong, China. ${ }^{8}$ School of Physics and Telecommunication Engineering, South China Normal University, Guangzhou, 510006 Guangdong, China. ${ }^{9}$ Interdisciplinary Institute of Light-Element Quantum Materials and Research Centre for Light-Element Advanced Materials, Peking University, 100871 Beijing, China. ${ }^{10}$ Shenzhen Institute for Quantum Science and Engineering, Southern University of Science and Technology, Shenzhen 518055 Guangdong, China. ${ }^{11}$ School of Physics, Liaoning University, Liaoning 110136 Shenyang, China. ${ }^{12}$ These authors contributed

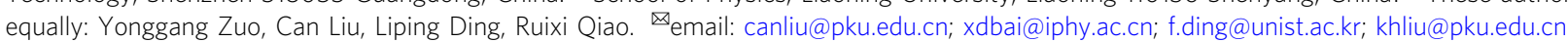


T wo-dimensional (2D) transition metal dichalcogenides (TMDs), with their atomic thicknesses, high carrier mobility, fast charge transfer, and intrinsic spin-valley couplings, have been demonstrated one of the most appealing candidates for next-generation electronic and optoelectronic devices ${ }^{1-3}$. The wafer-scale synthesis of TMDs with wellcontrolled crystallinity, quality, and composition is essential to fully realize their promising applications ${ }^{4-13}$. However, it is well known that the controllable growth of multi-element bulk materials is generally much more challenging than that of singleelement ones. For example, the synthesized first-generation semiconductor, silicon, can have the extremely low impurity of $\sim 10^{-11}$ and is nearly threading dislocation free, but the synthesized third-generation semiconductor, GaN, generally has a much higher impurity, $\sim 10^{-4}$, and a threading dislocation density of $\sim 10^{4}-10^{5} \mathrm{~cm}^{-2}$ (ref. ${ }^{14}$ ). Analogously for the growth of $2 \mathrm{D}$ materials, the as-grown graphene already has excellent properties, which is comparable to the samples exfoliated from natural crystals, and the measured carrier mobilities are close to the theoretical limit ${ }^{15}$, while the as-grown $2 \mathrm{D}$ compounds of TMDs, typically have lower quality than the natural crystals or the theoretical expectations $\mathrm{s}^{16}$. The main difficulty in controllable compounds' growth lies in the complicated feeding of several elements simultaneously during the growth process. Therefore, in the semiconductor industry, advanced and expensive techniques such as molecular beam epitaxy (MBE) and metal-organic chemical vapour deposition (MOCVD) have been developed to realize the precise control of multi-element supplies for the compound film growth.

Similarly, the synthesis of high-quality TMD materials requires the precise feeding control of both the transition metal and chalcogen precursors. In the past decade, intensive efforts have been devoted to optimizing the feeding of metal precursors by thermal evapouration or molten-salt-assisted evapouration of metal oxide ${ }^{17}$, decomposition of metal-organic precursor ${ }^{18}$, direct deposition of metal layers, and others ${ }^{19-21}$. Although some methods for controllable chalcogen feeding, such as using either elemental chalcogen or chalcogen compounds (i.e., heating sulfur powder, using $\mathrm{H}_{2} \mathrm{~S}$ gas and ammonium sulfide ${ }^{22-24}$ ), have also been developed, it turns out that the chalcogen feeding control is much less effective than metal feeding control, as indicated by the most challenging problem in TMD quality control that the most synthesized TMDs are rich with chalcogen vacancies ${ }^{25}$. Therefore, developing more effective chalcogen supply methods to enable the growth of TMDs with high quality and rich composition is of critical importance.

In this work, we propose to use a chalcogen monomer feeding method in the controllable TMD growth because of the following potential advantages. (i) The chalcogen monomers or atoms are generally very active than the corresponding dimers or bulks and thus they can quickly react with metal precursors to form TMDs, (ii) the active chalcogen monomers can bind and quickly diffuse on a TMD surface to scavenge the vacancy defects effectively, which will greatly improve the quality of the TMDs and (iii) an active chalcogen monomer can react with a TMD and easily substitute a chalcogen atom and, thus, allow the synthesis of uniform TMD chalcogen alloys. However, as the monomer state of chalcogen only exists at very high temperature $(>2500 \mathrm{~K}$ ) under normal circumstance ${ }^{26}$, the most used methods can not produce enough chalcogen monomers at the typical TMD growth temperature, which is generally $<\sim 1300 \mathrm{~K}$. Herein, we developed an effective route to provide chalcogen monomer by heating a metal chalcogenide. The success of this approach lies in that the chalcogen atoms on the surface of metal chalcogenides can be easily released in the chalcogen atom (monomer) form under a relatively lower temperature ${ }^{27,28}$. The slowly released chalcogen monomers have a very low probability to react with each other to form dimers, thus, enable the successful synthesis of TMDs $\left(\mathrm{MX}_{2}\right.$, $\mathrm{M}=\mathrm{Mo}, \mathrm{W} ; \mathrm{X}=\mathrm{S}, \mathrm{Se}, \mathrm{Te}$ ) and their alloys with very high quality.

\section{Results}

$\mathrm{MoS}_{2}$ growth by sulfur monomer supply. In our design, the metal chalcogenide plate of $\mathrm{ZnS}, \mathrm{Na}_{2} \mathrm{MoO}_{4}$ coated silica fibre fabric and the target substrate were vertically stacked as a sandwich structure by using mica spacers (Fig. 1a, left panel). The distance of $d_{1}\left(d_{2}\right)$ between the $\mathrm{S}(\mathrm{Mo})$ precursor and the growth substrate can be modulated by varying the thickness of mica (from tens to hundreds of microns), which is essential to tune the fluxes of $S$ and Mo independently. At an elevated growth temperature $\left(\sim 750-950^{\circ} \mathrm{C}\right), \mathrm{S}$ monomers were released from the $\mathrm{ZnS}$ surface and $\mathrm{Na}_{2} \mathrm{MoO}_{4}$ started to vapourize (Fig. 1a, middle panel). Then both $\mathrm{S}$ and Mo sources passed through the porous fibre fabric (Supplementary Fig. 1) and reached to the substrate surface to form monolayer $\mathrm{MoS}_{2}$ (Fig. 1a, right panel). It is important to note that the $S$ monomers can't be obtained by the sublimation of sulfur powders, where $S_{2}$ dimers are always the dominating species at thermal equilibrium ${ }^{29}$. Indeed, the release of $\mathrm{S}$ monomers from the metal sulfide surface was observed long time ago ${ }^{30}$, and was unambiguously revealed by the in-situ mass spectroscopy as shown in Fig. 1 b.

In our experiment, we found that the flux of $S$ monomers is significantly larger than that of the $\mathrm{Na}_{2} \mathrm{MoO}_{4}$ or the $\mathrm{S}$ is overfed (Fig. 1c and Supplementary Note 1), as we observed that the variation of $d_{1}$ (the distance between the $\mathrm{ZnS}$ and the substrate) has limited effect on the $\mathrm{MoS}_{2}$ growth, while the variation of $d_{2}$ (the distance between the fibre fabric and the substrate) affects the nucleation density of $\mathrm{MoS}_{2}$ domains greatly (Supplementary Fig. 2). This phenomenon can be qualitatively understood as that, by decreasing the $d_{2}$, the landing probability of evaporated Mo source onto the substrate becomes larger (Fig. 1d and Supplementary Note 2), which can further boost the nucleation of $\mathrm{MoS}_{2}$ domains. As shown in Fig. 1e, f, sparse triangular domains and 2-inch continuous film of $\mathrm{MoS}_{2}$ can be respectively obtained after the same growth duration of $50 \mathrm{~min}$ when $d_{2}$ was $100 \mu \mathrm{m}$ and $20 \mu \mathrm{m}$. In the limit case, the $d_{2}$ can also be zero by directly precoating a thin layer of $\mathrm{Na}_{2} \mathrm{MoO}_{4}$ precursor into the substrate (Supplementary Fig. 3).

Quality characterizations of $\mathrm{MoS}_{2}$ domains. A series of characterizations unequivocally reveal the high quality of $\mathrm{MoS}_{2}$ fed by $S$ monomer supply. Atomic-resolved atomic force microscopic (AFM) images at different positions of the as-grown $\mathrm{MoS}_{2}$ clearly resolved the $\mathrm{S}$ atoms without obvious $\mathrm{S}$ vacancies (Supplementary Fig. 4). High-angle annular dark-field scanning transmission electron microscopic (HAADF-STEM) images exhibited the perfect hexagonal honeycomb lattice with both Mo and $\mathrm{S}$ atoms (Fig. 2a and Supplementary Fig. 5). And the density of S vacancy defects was extracted as $\sim 2 \times 10^{12} \mathrm{~cm}^{-2}$, which is among the lowest value in previous STEM measurements (Supplementary Table 1).

The low-temperature photoluminescence (PL) spectra of the Smonomer-feeding-grown $\mathrm{MoS}_{2}$ (Fig. 2b, orange curve) has a characteristic neutral exciton $\left(\mathrm{X}^{0}\right)$ emission peak accompanied with a trion $\left(\mathrm{X}^{\mathrm{T}}\right)$ peak (believed to be caused by the n-type doping from substrate ${ }^{31}$ ), but the $\mathrm{X}^{\mathrm{D}}$ peak (believed to be caused by $\mathrm{S}$ vacancy ${ }^{32}$ and was obvious in S-powder-feeding-grown samples, Fig. 2b, dark yellow curve) is nearly invisible, which clearly proves the high quality of the S-monomer-feeding-grown $\mathrm{MoS}_{2}$ samples. Meanwhile, the uniform distribution of the PL peak intensity and the narrow full width at half maximum both 

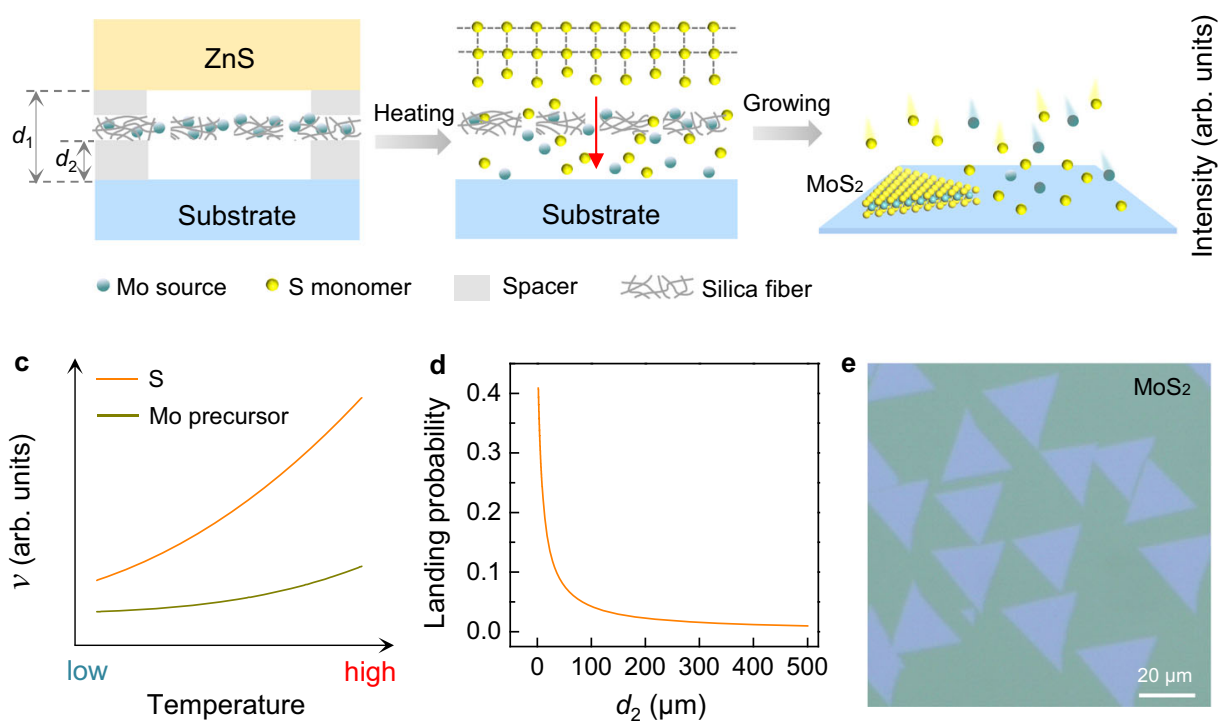
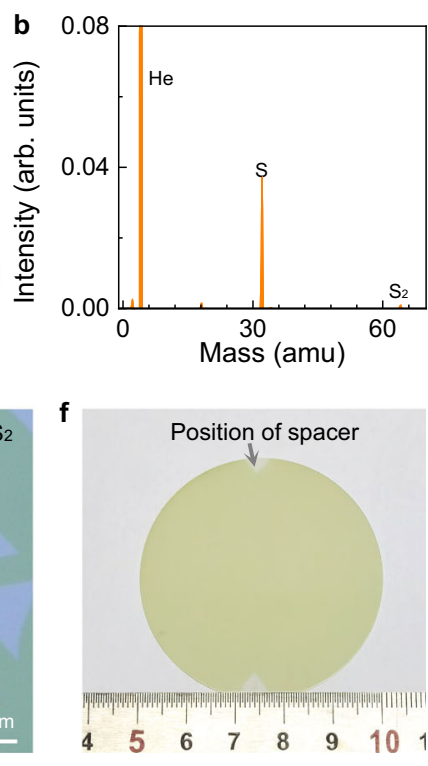

Fig. 1 Growth of wafer-scale monolayer $\mathbf{M o S}_{\mathbf{2}}$ by sulfur monomer supply. a Schematic of sulfur (S) monomer supply for the growth of MoS ${ }_{2}$. At high temperature, the released S monomers from the surface of $\mathrm{ZnS}$ and the vaporized Mo source from the precoated silica fiber fabric can penetrate the porous fabric and form monolayer $\mathrm{MoS}_{2}$ on the target substrate. $d_{1}$ and $d_{2}$ are the distances from the substrate to the $Z n S$ and the silica fiber fabric, respectively. The red arrow denotes the diffusion of $\mathrm{S}$ monomers released from the $\mathrm{ZnS}$ surface. $\mathbf{b}$ In-situ mass spectrum of $\mathrm{ZnS}$ annealed at $1000^{\circ} \mathrm{C}$. The intense peak at the mass of 32 clearly proves the dominating release of $\mathrm{S}$ monomers. The measurements were carried out with carrier gas of He and the data was subtracted by background. c The illustration of the temperature-dependent release rate of $\mathrm{S}$ monomers (orange curve) and evapouration rate of Mo precursor (dark yellow curve). d The landing probability of Mo precursor (orange curve) as a function of $d_{2}$ as modelled in Supplementary Note 2. e Optical image of the as-grown monolayer $\mathrm{MoS}_{2}$ domains on sapphire when $d_{2}$ is $100 \mu \mathrm{m}$. $\mathbf{f}$ Photograph of a 2-inch monolayer $\mathrm{MoS}_{2}$ film on sapphire when $d_{2}$ is $20 \mu \mathrm{m}$. The uncovered regions are the positions of the mica spacers.

demonstrated the high uniformity and crystallinity of the sample grown by $\mathrm{S}$ monomer supply (Supplementary Fig. 6).

In addition, the high quality of as-grown $\mathrm{MoS}_{2}$ can be further confirmed by the measured optical circular helicity ${ }^{33}$, which was detected to be as high as $92 \%$ (Fig. 2c) and comparable with the best-exfoliated flakes from high-quality natural crystals. The circular helicity is directly related to the scattering between $\mathrm{K}$ and $\mathrm{K}^{\prime}$ valleys in the Brillouin zone of $\mathrm{MoS}_{2}$ whilst the defects will greatly enhance the inter-valley scattering and decrease the circular helicity value. Thus, the near-unity circular helicity strongly proves the high quality of the as-grown $\mathrm{MoS}_{2}$ samples.

We further verified the quality of the single-crystal $\mathrm{MoS}_{2}$ domain by evaluating its field-effect mobility in a bottom-gate transistor configuration. The output and transfer characteristics of a typical field-effect transistor (FET) devices with channel length/width (L/W) of $7 / 22 \mu \mathrm{m}$ are exhibited in Fig. $2 \mathrm{~d}$ and Supplementary Fig. 7. The device exhibits a typical n-type transfer characteristic with an on/off ratio of $\sim 10^{8}$ at room temperature and electron field-effect mobilities of $\sim 42 \mathrm{~cm}^{2} \mathrm{~V}^{-1} \mathrm{~s}^{-1}$, which are comparable to the performance of monolayer $\mathrm{MoS}_{2}$ prepared by mechanical exfoliation ${ }^{34}$. Statistics of the transport measurement are also given in Supplementary Fig. 8, demonstrating the good uniformity of the $\mathrm{MoS}_{2}$ electronic devices. These results suggest that S-monomer-feeding-grown $\mathrm{MoS}_{2}$ samples have appreciably high electronic quality.

Universal TMD growth by chalcogen monomer supply. Our strategy on $\mathrm{MoS}_{2}$ growth by monomer feeding has also been proved to be applicable for the growth of various high-quality TMD materials. Six typical monolayer TMDs (Fig. 2e) have been successfully synthesized by simply replacing the transition metal sources (e.g., $\mathrm{Na}_{2} \mathrm{MoO}_{4}$ and $\mathrm{Na}_{2} \mathrm{WO}_{4}$ ) and chalcogenide plates (e.g., ZnS, ZnSe, and ZnTe) (see Methods and Supplementary
Fig. 9). The Raman and PL spectra of these obtained TMD samples demonstrated the successful synthesis of the $2 \mathrm{H}$ phase $\mathrm{MoS}_{2}, \mathrm{WS}_{2}, \mathrm{MoSe}_{2}, \mathrm{WSe}_{2}, \mathrm{MoTe}_{2}$, and the 1T' phase $\mathrm{WTe}_{2}$ and $\mathrm{MoTe}_{2}$ (Supplementary Fig. 10). It is worth noting that, due to their higher formation energies in relative to the corresponding sulfide and selenide bulks (Fig. 2 f and Supplementary Note 3), the growth of transition metal tellurides, e.g., $\mathrm{MoTe}_{2}$ and $\mathrm{WTe}_{2}$, by using chalcogen bulks as feedstocks is usually very challenging ${ }^{35}$. Thanks to the introduction of active Te monomers, the synthesis of $\mathrm{WTe}_{2}$ and $\mathrm{MoTe}_{2}$ is very easy and efficient because of the greatly reduced formation energies (Fig. 2f).

Controllable synthesis of quaternary TMD alloy. The chalcogen monomer feeding method has a unique advantage in the growth of TMD chalcogen alloys. Since the evapouration temperatures, saturated vapour pressures and reaction energies of $\mathrm{S}, \mathrm{Se}$, and $\mathrm{Te}$ are significantly different, it is nearly impossible to form highquality TMD alloys with more than two anion elements by traditional CVD approaches ${ }^{36}$. Till now, there is no report on the successful growth of $\mathrm{MoS}_{2(1-x-y)} \mathrm{Se}_{2 x} \mathrm{Te}_{2 y}$ alloy. In our experiment, we applied a compressed plate mixed with different metal chalcogenide powders, i.e., $\mathrm{ZnS}$, $\mathrm{ZnSe}$, and $\mathrm{ZnTe}$, to supply three kinds of chalcogen monomers ( $\mathrm{S}, \mathrm{Se}$, and $\mathrm{Te}$ ) simultaneously (Fig. 3a). The as-grown alloy of $\mathrm{MoS}_{2(1-x-y)} \mathrm{Se}_{2 x} \mathrm{Te}_{2 y}$ has a triangular domain similar to $2 \mathrm{H}$ phase TMDs (Fig. $3 \mathrm{~b}$ ). The X-ray photoelectron spectroscopy (XPS) unambiguously revealed the coexistence of $\mathrm{S}, \mathrm{Se}$, and Te atoms in the synthesized TMD alloy (Supplementary Fig. 11). The energy-dispersive X-ray spectroscopy (EDS) as well as the STEM measurements further demonstrated the homogenous element distribution throughout the TMD alloy in both macro- and micro-scales, with no observable phase separation (Fig. $3 \mathrm{e}$ and Supplementary Fig. 12). Enlarged STEM image (Fig. 3f) further demonstrated the high 

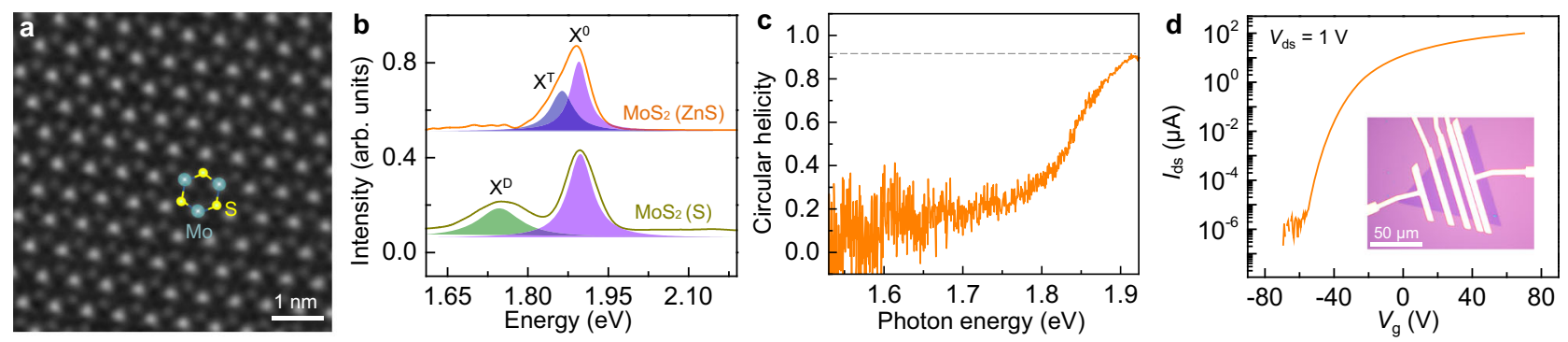
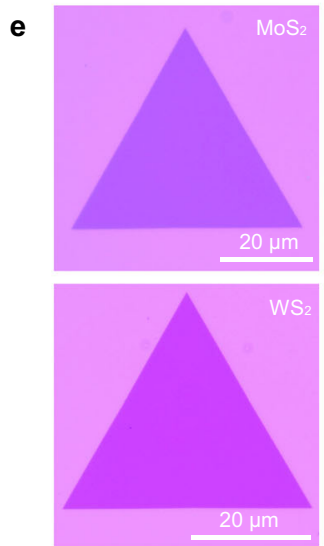
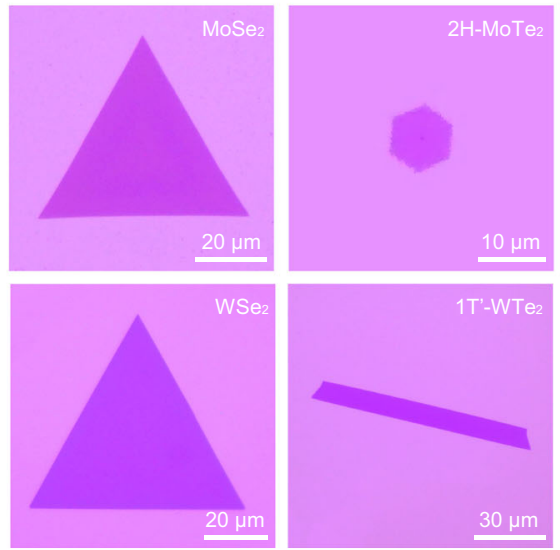

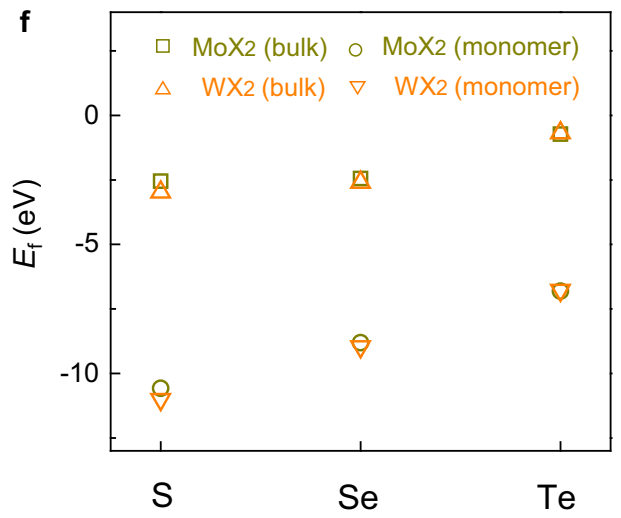

Fig. 2 Quality characterizations of the as-grown $\mathrm{MoS}_{\mathbf{2}}$ domain and universal growth of diverse TMDs by chalcogen monomer supply. a Atomicresolved HAADF-STEM image of the prepared $\mathrm{MoS}_{2}$, revealing the high crystallinity of $\mathrm{MoS}_{2}$ without detectable $\mathrm{S}$ vacancies. $\mathbf{b}$ Low-temperature (10 K) PL spectra of $\mathrm{MoS}_{2}$ samples fed by $\mathrm{S}$ monomer (orange curve) and $\mathrm{S}$ powder (dark yellow curve), respectively. Three typical features, $X^{0}, X^{\top}$, and $X^{D}$ peaks assign to neutral exciton, trion, and defect state emission peaks, respectively. The absence of $X^{D}$ peak confirmed the high quality of the MoS $S_{2}$ grown by $S$ monomer supply. c The circular dichroism PL spectrum measured at $10 \mathrm{~K}$. The near-unity polarization of $\mathrm{MoS}_{2}$ on sapphire indicates the high optical quality. The horizontal dashed line is added for clarity. d Transfer characteristic of the $\mathrm{MoS}_{2}$ FET with channel length and width of $7 \mu \mathrm{m}$ and $22 \mu \mathrm{m}$, respectively, at a bias voltage $V_{\text {ds }}$ of $1 \mathrm{~V}$. Inset: optical image of the device. e Optical images of the representative TMDs, including $2 \mathrm{H}$ phase $\mathrm{MoS}_{2}$, MoSe $\mathrm{C}_{2}$ $\mathrm{MoTe}_{2}, \mathrm{WS}_{2}, \mathrm{WSe}_{2}$ and $1 \mathrm{~T}^{\prime}$ phase $\mathrm{WTe}_{2}$. $\mathbf{f}$ The calculated formation energy $\left(E_{f}\right)$ of the six representative TMDs. When chalcogen bulks are supplied as precursors, the formation of transition metal tellurides in relative to their corresponding sulfide and selenide are less favourable due to their high formation energies ( -0.73 and $-0.68 \mathrm{eV} /$ unit for $\mathrm{MoTe}_{2}$ and $\mathrm{WTe}_{2}$, respectively). While it becomes highly favourable when Te monomers are applied.

crystallinity of the TMD alloy. The distinct intensity distribution revealed the occupancies of Mo, S, Se, and Te according to the Z-contrast nature of HAADF image (Fig. 3g). Quantitative analysis of the Te and Se distribution is presented in a $32 \times 32 \mathrm{~nm}^{2}$ STEM image, and the statistical results match well with the binomial distribution model (Fig. 3h, i, Supplementary Fig. 13, and Supplementary Note 4), suggesting a random distribution of $\mathrm{S}$, Se and Te atoms in the TMD alloy.

Furthermore, one can easily tune the composition of the TMD alloys by controlling the growth temperature to vary the fluxes of $\mathrm{S}$, Se, and Te from the $\mathrm{ZnS}, \mathrm{ZnSe}$, and $\mathrm{ZnTe}$ composite. As the growth temperature increased, the PL peak shifted to longer wavelength (Fig. 3c), revealing the increase of the concentrations of Se and $\mathrm{Te}$ in the alloy $\left(\mathrm{MoSe}_{2}\right.$ and $\mathrm{MoTe}_{2}$ have smaller bandgaps than $\mathrm{MoS}_{2}$ ). Meanwhile, in the Raman spectra, the $\mathrm{MoS}_{2}$-featured peaks gradually attenuated, the $\mathrm{MoTe}_{2}$-featured peaks gradually enhanced, while the $\mathrm{MoSe}_{2}$-featured peaks enhanced first and then attenuated (Fig. 3d), which indicates that heavier chalcogen atoms are being doped into the alloy at a higher temperature.

Mechanism for the chalcogen monomer-modulated TMD growth. Finally, we try to understand the unique role of chalcogen monomer supply in the synthesis of high-quality TMDs and their complex alloys theoretically. We firstly explore the reactions of $\mathrm{Na}_{2} \mathrm{MoO}_{4}$ with sulfur monomers and dimers, respectively, by first-principles molecular dynamic (MD) simulations (Supplementary Fig. 14). The simulation results clearly demonstrated that sulfur monomers are more reactive to substitute the oxygen atoms in a $\mathrm{MoO}_{4}{ }^{2-}$ group of $\mathrm{Na}_{2} \mathrm{MoO}_{4}$ while most dimers are desorbed from the $\mathrm{Na}_{2} \mathrm{MoO}_{4}$ surface due to their less activity (Supplementary Note 5 ). By adding more $\mathrm{S}$ monomers and $\mathrm{MoS}_{3}$ clusters to the $\mathrm{Na}_{2} \mathrm{MoO}_{4}$, the nucleation of $\mathrm{Mo}_{x} \mathrm{~S}_{y}$ clusters can also be clearly seen (Supplementary Fig. 15).

In compare with the bond-saturated $\mathrm{S}_{2} / \mathrm{Se}_{2} / \mathrm{Te}_{2}$ dimers, $\mathrm{S} / \mathrm{Se} / \mathrm{Te}$ monomers possess much higher adsorption energies on a TMD surface (for dimers $E_{\mathrm{b}}<0.8 \mathrm{eV}$, for monomers $E_{\mathrm{b}}>1.5 \mathrm{eV}$ ) (Fig. $4 \mathrm{~b}$ and Supplementary Fig. 16a). Therefore, one can expect a large number of S/Se/Te monomers to diffuse on the TMD surface during the whole growth process and the growth of TMD is in a chalcogen monomer rich environment. Once a chalcogen monomer diffuses to the vicinal area of a vacancy, the vacancy can be quickly healed by a highly exothermic reaction (Fig. 4a, c and Supplementary Fig. 16b). Therefore, the chalcogen vacancy density is greatly reduced as has been shown in the experimental results. To address the capacity of forming TMD chalcogen alloys, we calculated the reaction energy of substituting a chalcogen atom in a TMD by using chalcogen monomer, dimer, and bulk as references (Supplementary Fig. 17 and Supplementary Note 6). It is clearly shown that doping of Te dimer or bulk into $\mathrm{MoS}_{2}$ / $\mathrm{MoSe}_{2}$, S bulk into $\mathrm{MoSe}_{2}$, Se bulk into $\mathrm{MoS}_{2}$ are all difficult because of the near-zero or positive reaction energies. While, if chalcogen monomer is used as the source of dopant, all doping reactions become exothermic with noticeable negative reaction energies, which implies the greatly improved capacity of forming chalcogen TMD alloys. 

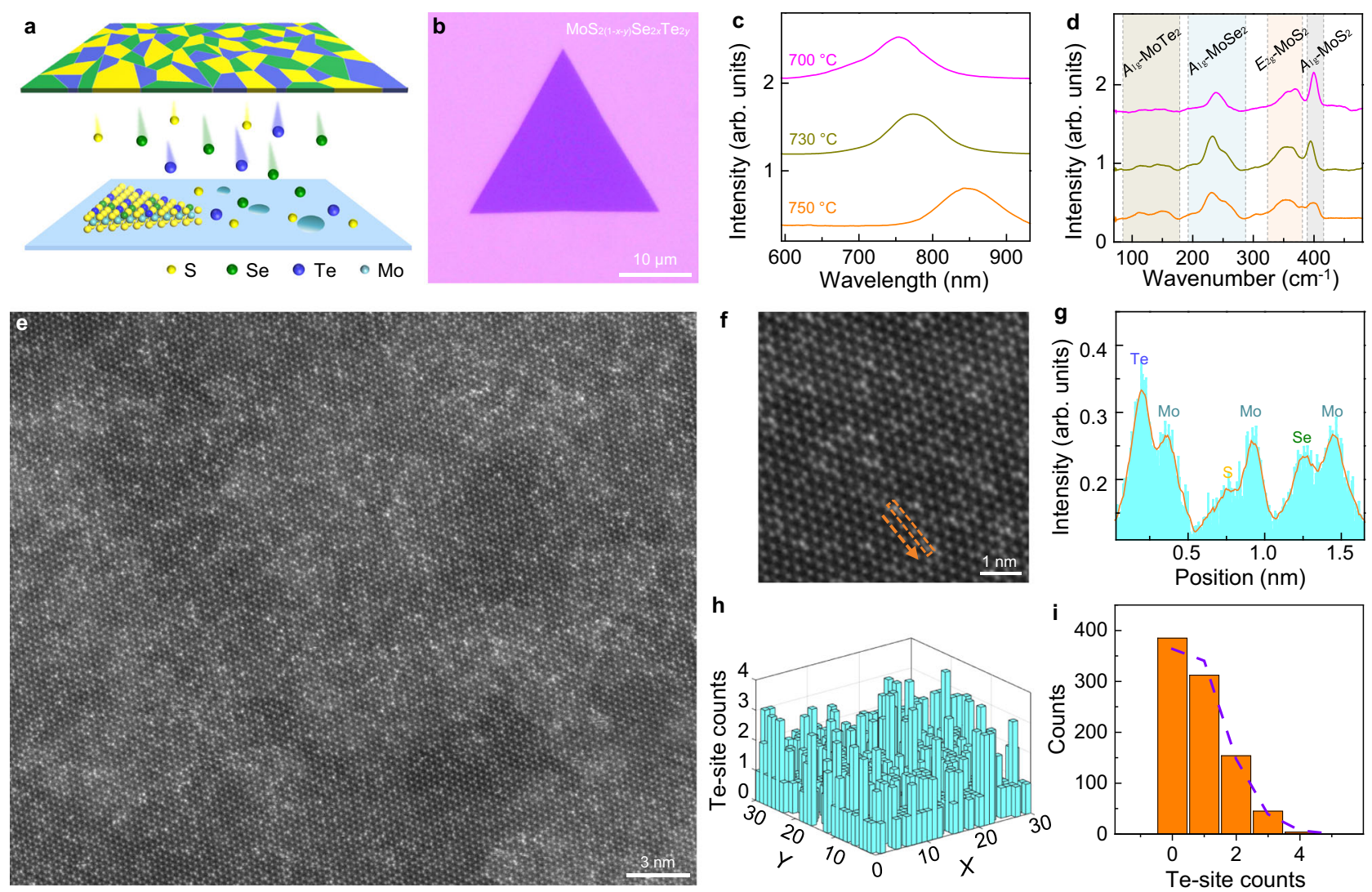

Fig. 3 Growth and characterizations of quaternary TMD alloy. a Schematic diagram of quaternary alloy growth using a compressed plate mixed with chalcogenide powders of $\mathrm{ZnS}, \mathrm{ZnSe}$, and $\mathrm{ZnTe}$. b Optical image of $\mathrm{MoS}_{2(1-x-y)} \mathrm{Se}_{2 x} \mathrm{Te}_{2 y}$ domain on $\mathrm{SiO}_{2} / \mathrm{Si}$ substrate. c, d PL (c) and Raman (d) spectra of the $\mathrm{MoS}_{2(1-x-y)} \mathrm{Se}_{2 x} \mathrm{Te}_{2 y}$ sample grown at different temperatures. As the growth temperature increased, the PL peak position showed a clear red shift. The intensity of $\mathrm{MoS}_{2}$-like $E_{2 \mathrm{~g}}\left(\sim 380 \mathrm{~cm}^{-1}\right)$ and $A_{1 \mathrm{~g}}\left(\sim 400 \mathrm{~cm}^{-1}\right)$ was reduced while the MoTe-like $A_{1 \mathrm{~g}}\left(\sim 150 \mathrm{~cm}^{-1}\right)$ increased and MoSe $\mathrm{Me}_{2}$-like $A_{1 \mathrm{~g}}\left(\sim 240 \mathrm{~cm}^{-1}\right)$ increased first and then reduced. The shaded areas are added for clarity. e, f STEM images of the $\mathrm{MoS}_{2(1-x-y)} \mathrm{Se}_{2 x} \mathrm{Te}_{2 y}$, demonstrating the high crystallinity of quaternary alloy. $\mathbf{g}$ Intensity profile along the labeled orange dotted box in (f), which highlights the occupancies of Mo, S, Se, and Te sites. $\mathbf{h}$ The Te-site distribution in a $32 \times 32 \mathrm{~nm}^{2}$ STEM image of the quaternary alloy. The image was divided into $30 \times 30$ parts. $\mathbf{i}$ The corresponding statistical histogram of Te-site counts in each parts of the image. It shows a well binomial distribution feature (purple dotted line), revealing the random distribution of Te atoms.

\section{Discussion}

This study clearly demonstrated that the high reactivity of chalcogen monomers can significantly facilitate the TMD nucleation, chalcogen defect healing in the growth to greatly improve the samples' quality and allow the formation of various chalcogen TMDs and their alloys. The monomer supply should provide a degree of freedom in modulating compound materials and highentropy $2 \mathrm{D}$ alloys, thus widening their potential applications in electronic, optoelectronic and valleytronic devices.

\section{Methods}

Growth of TMDs and their alloys. The substrate (sapphire or $\mathrm{SiO}_{2} / \mathrm{Si}$ ) and silica fibre fabric were first pretreated with $\mathrm{O}_{2}$ plasma. Then the silica fibre fabric was immersed in $\mathrm{Na}_{2} \mathrm{MoO}_{4}$ or $\mathrm{Na}_{2} \mathrm{WO}_{4}$ aqueous solution with optimized concentrations $\left(\mathrm{Na}_{2} \mathrm{MoO}_{4}\right.$ of 12,18 , and $6 \mathrm{mg} / \mathrm{mL}$ for $\mathrm{MoS}_{2}, \mathrm{MoSe}_{2}$, and $\mathrm{MoTe}_{2}$ growth, $\mathrm{Na}_{2} \mathrm{WO}_{4}$ of 20,30 , and $12 \mathrm{mg} / \mathrm{mL}$ for $\mathrm{WS}_{2}, \mathrm{WSe}_{2}$, and $\mathrm{WTe}_{2}$ growth, respectively). After dried in $\mathrm{Ar}$ atmosphere, silica fibre fabric and the chalcogenide crystal plate ( $\mathrm{ZnS}, \mathrm{ZnSe}$, or $\mathrm{ZnTe}$ ) were placed above the substrate in sequence by using two pieces of mica as the spacers respectively, and then loaded into the CVD furnace together. The chamber of furnace was flushed with $\operatorname{Ar}(100 \mathrm{sccm})$ and heated to the optimized growth temperature $\left(\sim 780^{\circ} \mathrm{C}, \sim 930^{\circ} \mathrm{C}, \sim 800^{\circ} \mathrm{C}, \sim 820^{\circ} \mathrm{C}, \sim 750-800^{\circ} \mathrm{C}\right.$, $\sim 800^{\circ} \mathrm{C}$, and $\sim 780^{\circ} \mathrm{C}$ for $\mathrm{MoS}_{2}, \mathrm{WS}_{2}, \mathrm{MoSe}_{2}, \mathrm{WSe} \mathrm{H}_{2}, 2 \mathrm{H}-\mathrm{MoTe}_{2}, 1 \mathrm{~T}^{\prime}-\mathrm{MoTe}_{2}$, and $1 \mathrm{~T}^{\prime}-\mathrm{WTe}_{2}$, respectively). During the growth process, the system pressure was kept at $\sim 120 \mathrm{~Pa}$ and the growth duration was set as $10-60 \mathrm{~min}$. After growth, the system was naturally cooled down to room temperature. Similar growth conditions were applied to the TMD alloy growth, wherein the major difference lies in the use of a chalcogenide mixture plate.
Sample characterizations. Optical images were taken with an Olympus BX51M microscope. Raman and PL spectra were measured by a customer-designed optical system with the excitation wavelength of $532 \mathrm{~nm}$ and the power of $\sim 1 \mathrm{~mW}$. Lowtemperature PL spectra were obtained at $10 \mathrm{~K}$ by optical cryostat (Montana Instruments) with the laser excitation wavelength of $532 \mathrm{~nm}$. Circular-polarizationresolved PL measurements were performed under near-resonant excitation of $633 \mathrm{~nm}$ at $10 \mathrm{~K}$. The circularly polarized light was generated by using a superachromatic quarter-wave plate (Thorlabs SAQWP05M-1700) and the photoluminescence was analyzed through the same quarter-wave plate and a linearpolarizer. We define the degree of PL circular helicity $(\eta)$, which reflects the valley polarization, as $\eta=\left[\mathrm{PL}\left(\sigma^{+}\right)-\mathrm{PL}\left(\sigma^{-}\right)\right] /\left[\mathrm{PL}\left(\sigma^{+}\right)+\mathrm{PL}\left(\sigma^{-}\right)\right]$. XPS measurements were performed using an ESCALAB 250X system (Thermo Fisher Scientific) and excited by monochromatic $\mathrm{Al} \mathrm{Ka}$ radiation. Mass spectrometer (Hiden HR20) attached with temperature-programmed decomposition (TPD) was used to in-situ detect and analyze the released gas in inert atmosphere. EDS and STEM experiments were performed in FEI Titan Themis G2 300 operated at $300 \mathrm{kV}$ and in Nion U-HERMES200 at $60 \mathrm{kV}$ for element analysis and characterizing atomic structures of samples. Atomic-resolved AFM measurements were performed using Asylum Research Cypher in ambient atmosphere.

Fabrication and measurement of $\mathbf{M o S}_{\mathbf{2}}$ FET device. Electron-beam lithography (EBL) is used to define the channel and the source/drain contacts with PMMA EBL resists. Metallization is implemented by thermal evapouration of $20 \mathrm{~nm}$ bismuth with a rate of $0.2 \AA \mathrm{s}^{-1}$, followed by an Au capping layer by electron-beam evapouration $\left(30 \mathrm{~nm}\right.$ at $0.1 \AA^{-1}$ ) at $\sim 10^{-7}$ torr. Lift-off process is carried out in hot acetone. All electrical characterization is conducted in a vacuum environment and room temperature in a Janis probe station using a semiconductor device analyser (Agilent Technologies B1500A). The field mobilities of $\mathrm{MoS}_{2}$ were calculated according to the equation $\mu=\left[\mathrm{d} I_{\mathrm{ds}} / \mathrm{d} V_{\mathrm{g}}\right] \times\left[L /\left(W C_{\mathrm{i}} V_{\mathrm{ds}}\right)\right]$. In this equation, the $L$ 

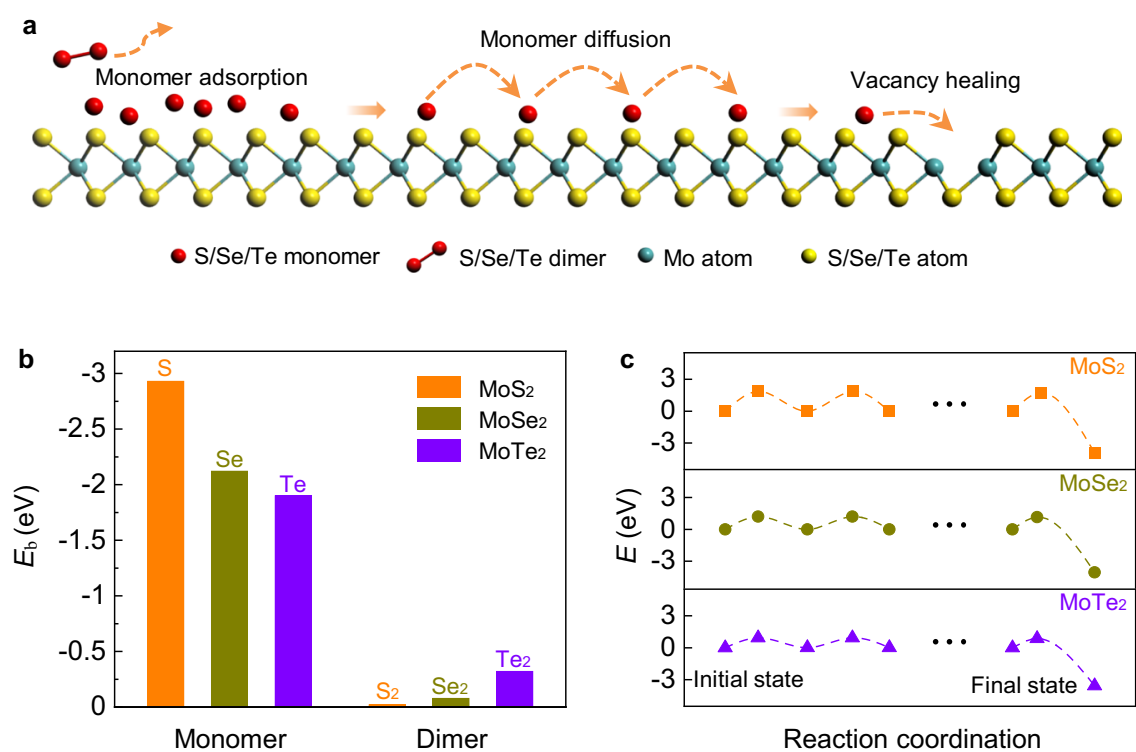

Fig. 4 Growth mechanism with chalcogen monomer supply in $\mathbf{M o X}_{\mathbf{2}}(\mathbf{X}=\mathbf{S}, \mathbf{S e}, \mathbf{T e})$. a Schematic diagram of adsorption, diffusion, and vacancy healing of chalcogen monomer on $\mathrm{MoX}_{2}$ surface. The orange dotted arrows denote the motion of chalcogen monomers or dimers. $\mathbf{b}$ The binding energies of monomers and dimers on $\mathrm{MoX}_{2}$ surface. The much higher energy of monomers facilitates their better adsorption on the TMD surface than dimers. $\mathbf{c}$ The energy profiles of vacancy healing for $\mathrm{MoX}_{2}$ surface by using chalcogen monomers. The relatively small energy barriers of chalcogen monomer diffusion and the highly exothermic reaction at the vacancy both accelerate the self-healing of MoX .

and $W$ are the channel length and width, respectively. $C_{\mathrm{i}}$ is the capacitance per unit area of $300 \mathrm{~nm} \mathrm{SiO}$ dielectric layer, $11.5 \times 10^{-5} \mathrm{~F}$

Computational details. All the density functional theory (DFT) calculations were implemented by the Vienna Ab Initio Simulation Package (VASP) ${ }^{37,38}$, with projector-augmented wave (PAW) ${ }^{39}$ method describing the interaction between valence electrons and ion cores. The Perdew-Burke-Ernzerhof $(\mathrm{PBE})^{40,41}$ exchange-correlation functional was used to describe the interaction between electrons. A plane wave basis set with a cutoff energy of $450 \mathrm{eV}$ was adopted. All the structures were fully relaxed, and the convergence criteria for energy and force were set at $10^{-5} \mathrm{eV}$ and $10^{-2} \mathrm{eV} / \AA$, respectively. The Brillion zone is sampled by $1 \times 1 \times 1$ grid meshes. A vacuum spacing larger than $15 \AA$ was set to avoid the interaction between neighbouring images along the non-periodic direction. The energy barriers were calculated by using the climbing image nudged elastic band (CI-NEB) method $^{42}$ with a force threshold of $-0.02 \mathrm{eV} / \AA$.

\section{Data availability}

The authors declare that the data supporting the findings of this study are available within the paper, Supplementary Information and Source Data. Extra data are available from the corresponding authors upon request. Source data are provided with this paper.

Received: 3 August 2021; Accepted: 26 January 2022;

Published online: 23 February 2022

\section{References}

1. Ye, Z. et al. Probing excitonic dark states in single-layer tungsten disulphide. Nature 513, 214-218 (2014).

2. Novoselov, K. S., Mishchenko, A., Carvalho, A. \& Castro Neto, A. H. 2D materials and van der Waals heterostructures. Science 353, ac9439 (2016).

3. Jauregui, L. A. et al. Electrical control of interlayer exciton dynamics in atomically thin heterostructures. Science 366, 870-875 (2019).

4. Chhowalla, M. et al. The chemistry of two-dimensional layered transition metal dichalcogenide nanosheets. Nat. Chem. 5, 263-275 (2013).

5. Najmaei, S. et al. Vapour phase growth and grain boundary structure of molybdenum disulphide atomic layers. Nat. Mater. 12, 754-759 (2013).

6. Van der Zande, A. M. et al. Grains and grain boundaries in highly crystalline monolayer molybdenum disulphide. Nat. Mater. 12, 554-561 (2013).

7. Huang, C. et al. Lateral heterojunctions within monolayer $\mathrm{MoSe}_{2}-\mathrm{WSe}_{2}$ semiconductors. Nat. Mater. 13, 1096-1101 (2014).
8. Li, M.-Y. et al. Epitaxial growth of a monolayer $\mathrm{WSe}_{2}-\mathrm{MoS}_{2}$ lateral p-n junction with an atomically sharp interface. Science 349, 524-528 (2015).

9. Li, S. et al. Vapour-liquid-solid growth of monolayer $\mathrm{MoS}_{2}$ nanoribbons. Nat. Mater. 17, 535-542 (2018).

10. Sahoo, P. K. et al. One-pot growth of two-dimensional lateral heterostructures via sequential edge-epitaxy. Nature 553, 63-67 (2018).

11. Aljarb, A. et al. Ledge-directed epitaxy of continuously self-aligned singlecrystalline nanoribbons of transition metal dichalcogenides. Nat. Mater. 19, 1300-1306 (2020)

12. Chowdhury, T. et al. Substrate-directed synthesis of $\mathrm{MoS}_{2}$ nanocrystals with tunable dimensionality and optical properties. Nat. Nanotechnol. 15, 29-34 (2020).

13. Zeng, M. et al. Bandgap tuning of two-dimensional materials by sphere diameter engineering. Nat. Mater. 19, 528-533 (2020).

14. Zajac, M. et al. Basic ammonothermal growth of gallium nitride-state of the art, challenges, perspectives. Prog. Cryst. Growth Charact. Mater. 64, 63-74 (2018).

15. Wang, L. et al. One-dimensional electrical contact to a two-dimensional material. Science 342, 614-617 (2013).

16. Kaasbjerg, K., Thygesen, K. S. \& Jacobsen, K. W. Phonon-limited mobility in n-type single-layer $\mathrm{MoS}_{2}$ from first principles. Phys. Rev. B 85, 115317 (2012).

17. Zhou, J. et al. A library of atomically thin metal chalcogenides. Nature 556, 355-359 (2018).

18. Kang, K. et al. High-mobility three-atom-thick semiconducting films with wafer-scale homogeneity. Nature 520, 656-660 (2015).

19. Liu, L. et al. Phase-selective synthesis of $1 \mathrm{~T}^{\prime} \mathrm{MoS}_{2}$ monolayers and heterophase bilayers. Nat. Mater. 17, 1108-1114 (2018).

20. Shivayogimath, A. et al. A universal approach for the synthesis of twodimensional binary compounds. Nat. Commun. 10, 2957 (2019).

21. Li, N. et al. Large-scale flexible and transparent electronics based on monolayer molybdenum disulfide field-effect transistors. Nat. Electron. 3, 711-717 (2020).

22. Lin, H. et al. Growth of environmentally stable transition metal selenide films. Nat. Mater. 18, 602-607 (2019).

23. Dumcenco, D. et al. Large-area $\mathrm{MoS}_{2}$ grown using $\mathrm{H}_{2} \mathrm{~S}$ as the sulphur source. 2D Materials 2, 044005 (2015).

24. Lee, J. S. et al. Wafer-scale single-crystal hexagonal boron nitride film via selfcollimated grain formation. Science 362, 817-821 (2018).

25. Hong, J. et al. Exploring atomic defects in molybdenum disulphide monolayers. Nat. Commun. 6, 6293 (2015).

26. Berkowitz, J. \& Marquart, J. R. Equilibrium composition of sulfur vapor. J. Chem. Phys. 39, 275-283 (1963).

27. $\mathrm{Xu}, \mathrm{X}$. et al. Ultrafast growth of single-crystal graphene assisted by a continuous oxygen supply. Nat. Nanotechnol. 11, 930-935 (2016). 
28. Liu, C. et al. Kinetic modulation of graphene growth by fluorine through spatially confined decomposition of metal fluorides. Nat. Chem. 11, 730-736 (2019).

29. Steudel, R., Steudel, Y. \& Wong, M. W. Speciation and thermodynamics of sulfur vapor. Top. Curr. Chem. 230, 117-134 (2003).

30. Pulker, H. K. \& Jung, E. An investigation of the evaporation process of dielectric materials. Thin Solid Films 4, 219-228 (1969).

31. Mak, K. F. et al. Tightly bound trions in monolayer $\mathrm{MoS}_{2}$. Nat. Mater. 12, 207-211 (2013).

32. Carozo, V. et al. Optical identification of sulfur vacancies: Bound excitons at the edges of monolayer tungsten disulfide. Sci. Adv. 3, el602813 (2017)

33. Cao, T. et al. Valley-selective circular dichroism of monolayer molybdenum disulphide. Nat. Commun. 3, 887 (2012).

34. Radisavljevic, B. et al. Single-layer $\mathrm{MoS}_{2}$ transistors. Nat. Nanotechnol. 6, 147-150 (2011).

35. Sung, J. H. et al. Coplanar semiconductor-metal circuitry defined on few-layer $\mathrm{MoTe}_{2}$ via polymorphic heteroepitaxy. Nat. Nanotechnol. 12, 1064-1071 (2017).

36. Mann, J. et al. 2-Dimensional transition metal dichalcogenides with tunable direct band gaps: $\mathrm{MoS}_{2(1-\mathrm{x})} \mathrm{Se}_{2 \mathrm{x}}$ monolayers. Adv. Mater. 26, 1399-1404 (2014).

37. Kresse, G. \& Hafner, J. Ab-initio molecular-dynamics for open-shell transition-metals. Phys. Rev. B 48, 13115-13118 (1993).

38. Kresse, G. \& Furthmüller, J. Efficiency of ab-initio total energy calculations for metals and semiconductors using a plane-wave basis set. Comput. Mater. Sci. 6, 15-50 (1996).

39. Kresse, G. \& Furthmüller, J. Efficient iterative schemes for ab initio total-energy calculations using a plane-wave basis set. Phys. Rev. B 54, 11169-11186 (1996).

40. Perdew, J. P., Burke, K. \& Ernzerhof, M. Generalized gradient approximation made simple. Phys. Rev. Lett. 77, 3865-3868 (1996).

41. Blöchl, P. E. Projector augmented-wave method. Phys. Rev. B 50, 17953-17979 (1994).

42. Henkelman, G., Uberuaga, B. P. \& Jonsson, H. A climbing image nudged elastic band method for finding saddle points and minimum energy paths. $J$. Chem. Phys. 113, 9901-9904 (2000).

\section{Acknowledgements}

This work was supported by Guangdong Major Project of Basic and Applied Basic Research with grant number 2021B0301030002 (E.W. and Kaihui Liu); the National Key R\&D Program of China under grant numbers 2021YFA1400502 (M.W.) and 2021YFA1400201 (H.H.); the National Natural Science Foundation of China under grant numbers 52025023 (Kaihui Liu), 51991342 (Kaihui Liu), 52021006 (Kaihui Liu), 51991344 (X.B.), 11888101 (E.W.) and 92163206 (M.W.); the Key R\&D Program of Guangdong Province under grant numbers 2020B010189001 (Kaihui Liu), 2019B010931001 (Kaihui Liu) and 2018B030327001 (D.Y.); the Pearl River Talent Recruitment Program of Guangdong Province with grant number 2019ZT08C321 (Kaihui Liu); the Strategic Priority Research Program of Chinese Academy of Sciences under grant numbers XDB33000000 (Kaihui Liu) and XDB33030200
(X.B.). We acknowledge the Electron Microscopy Laboratory in Peking University for the use of electron microscopy.

\section{Author contributions}

Kaihui Liu, F.D., X.B., and Can Liu supervised the project. Y.Z. and Can Liu conducted the sample growth and characterizations. L.D. and F.D. performed the theoretical calculations. R.Q., Q.G., and X.B. performed the STEM experiments. J.T., R.Y., and G.Z. performed the electrical measurements. Chang Liu, X.Z., and H.H. executed the lowtemperature PL and valley polarization measurements. Q.W., G.X., Y.Y., J.W., Y.F., Kehai Liu, and M.W. helped sample characterizations. X.L. helped analysis electrical data. Y.Z. Can Liu, L.D., R.Q., Kaihui Liu, and F.D. wrote the article. X.B., D.Y., and E.W. revised the manuscript. All the authors discussed the results and commented on the manuscript.

\section{Competing interests}

The authors declare no completing interests.

\section{Additional information}

Supplementary information The online version contains supplementary material available at https://doi.org/10.1038/s41467-022-28628-7.

Correspondence and requests for materials should be addressed to Can Liu, Xuedong Bai, Feng Ding or Kaihui Liu.

Peer review information Nature Communications thanks Po-Wen Chiu and the other anonymous reviewers for their contribution to the peer review of this work

Reprints and permission information is available at http://www.nature.com/reprints

Publisher's note Springer Nature remains neutral with regard to jurisdictional claims in published maps and institutional affiliations.

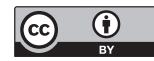

Open Access This article is licensed under a Creative Commons Attribution 4.0 International License, which permits use, sharing, adaptation, distribution and reproduction in any medium or format, as long as you give appropriate credit to the original author(s) and the source, provide a link to the Creative Commons license, and indicate if changes were made. The images or other third party material in this article are included in the article's Creative Commons license, unles indicated otherwise in a credit line to the material. If material is not included in the article's Creative Commons license and your intended use is not permitted by statutory regulation or exceeds the permitted use, you will need to obtain permission directly from the copyright holder. To view a copy of this license, visit http://creativecommons.org/ licenses/by/4.0/

(C) The Author(s) 2022 